\title{
Genital infection and termination of pregnancy: Are patients still at risk?
}

Caroline D Smith, MB BS, DFFP, Senior House Officer; Elizabeth M Carlin, DFFP, FRCP, Consultant; Judy Heason, BSc, Health Advisor, Department of Genitourinary Medicine, Nottingham City Hospital, Nottingham, UK

David TY Liu, MD, FRCOG, Consultant, Department of Obstetrics and Gynaecology, Nottingham City Hospital, Nottingham, UK Imtyaz Ahmed Jushuf, MB ChB, FRCP, Consultant, Department of Genitourinary Medicine, Nottingham City Hospital, Nottingham, UK

Robert H Hammond, FRCS, FRCOG, Consultant, Department of Obstetrics and Gynaecology, Queen's Medical Centre, Nottingham, UK

Correspondence: Dr EM Carlin, Consultant Physician, Department of Genitourinary Medicine, Nottingham City Hospital, Hucknall Road, Nottingham, NG5 1PB, UK. Tel: 0115962 7746, Fax: 01159627684

(Accepted November 21 $\left.1^{\text {st }}, 2000\right)$

\section{Summary}

Objectives. To identify the treatment patterns and early complication rates in women identified with a genital infection prior to a termination of pregnancy $(T O P)$ and to re-assess our earlier work on contact attendance and treatment. The aim was to refine current prophylactic measures to provide the most efficient and effective way of reducing post-termination complications.

Method. Six-month retrospective analysis of all women with genital Chlamydia trachomatis or Neisseria gonorrhoeae identified prior to TOP. Data on the timing of positive results and treatment in relation to the TOP were recorded. Clinical signs of pelvic infection and the success of contact tracing and treatment were noted.

Results. Forty women were identified with infection, 31 (78\%) proceeded to TOP; only five were treated adequately in the peri-TOP period. Twenty-six (84\%) of the women undergoing TOP attended the genitourinary medicine (GUM) department after the TOP and received antimicrobial therapy where necessary. In total, four women (10\%) were untreated. Approximately two thirds of results were reported prior to, or on the day of, the TOP. The majority of TOPs $(71 \%)$ were carried out at 5 days or less from the initial assessment. Clinical signs of post-TOP pelvic inflammation developed in seven (28\%) women with chlamydial infection. Contact tracing was successful in 24 (69\%) of the 35 women who attended the GUM department.

Conclusion. The debate about the best option for the most effective management of women undergoing TOP continues. The options with respect to $\mathrm{C}$. trachomatis include universal prophylaxis, screening and treating as necessary, or a 'belt and braces' approach of screening all and treating all. Fundamental is the continuation of active contact tracing and GUM remains integral to this.

Key words

Chlamydia trachomatis, termination of pregnancy, screening.

\section{Key message points}

- Surgical TOP is associated with a significant rate of early complications in the presence of untreated genital chlamydial infection.

- Many TOPs are performed rapidly after the initial assessment, hence the need for a rapid turn-round time for screening test results.

- For women in whom genital gonorrhoea or chlamydial infection is detected, contact tracing and treatment of sexual partners is essential.

\section{Introduction}

The risks of untreated genital infection in association with surgical termination of pregnancy (TOP), particularly with Chlamydia trachomatis and Neisseria gonorrhoeae, are well reported. Risks include pelvic inflammatory disease (PID), ectopic pregnancy, tubal factor infertility and chronic pelvic pain. ${ }^{1-5}$ The value of screening for genital tract infection is thus widely accepted. ${ }^{1,6}$ As the risk of upper genital tract infection is increased by instrumentation of the cervix, it seems important that treatment be given prior to, or at induction of, TOP. In addition, an integral part of management includes effective treatment of sexual partners in order to prevent re-infection and as a public health measure.

As a consequence, it is our practice in Nottingham to screen all women prior to TOP for genital infections including $C$. trachomatis and $N$. gonorrhoeae. We have previously reported on our local model of care for managing sexually transmitted infections identified in departments of obstetrics and gynaecology. ${ }^{7}$ In this model positive results for chlamydial and gonococcal infection are referred to the genitourinary medicine (GUM) department from the microbiology department. The women are then contacted directly by the department of GUM and offered a fast track appointment to ensure adequate treatment has been completed and to allow health education and contact tracing. ${ }^{7}$

Aims

To identify the treatment patterns and early complication rates in women identified with a genital infection prior to TOP and to re-assess our earlier work on contact attendance and treatment.

\section{Method}

A retrospective case note study was performed over a 6-month period of all women with positive $C$. trachomatis or $N$. gonorrhoeae results identified by enzyme-linked immunoassay (EIA) or culture, respectively. Screening was performed prior to TOP in the gynaecology out-patient department and GUM involvement was as described previously. ${ }^{7}$ Data were collected on: the timing of treatment and reporting of positive results in relation to the TOP; subsequent clinical signs of pelvic infection; contact tracing and treatment.

\section{Results}

During the 6 month period, 40 women were identified of which 38 were positive for $C$. trachomatis alone, one was 
positive for $C$. trachomatis and N. gonorrhoeae, and one was positive for $N$. gonorrhoeae alone.

Thirty-five women attended the GUM department, nine of whom did not proceed with the TOP. The five women who did not attend the GUM department all proceeded with the TOP. Of the 31 terminations performed, 29 were suction terminations and two were prostaglandin-induced, but required evacuation of retained products of conception. Eight of the women who decided to proceed with the pregnancy were positive for $C$. trachomatis and one had a dual infection with $C$. trachomatis and $N$. gonorrhoeae. Hence, $23 \%$ of women who attended a gynaecology outpatient clinic and were booked for a TOP decided not to proceed. No specific reasons were given by the women for this decision other than a desire to continue with the pregnancy.

Only two women out of the $31(6 \%)$ who proceeded to termination were treated adequately either before, or at induction, of TOP (Figure 1). One of those treated was done so incidentally as she required treatment for a Bartholin's abscess. A further three women were treated adequately on discharge, by the gynaecologists, following their TOP (Figure 1). Four of the five women who had already received antibiotic treatment via the gynaecologists attended the GUM department, one woman was from the pre-operative treatment group and the remainder had been treated on discharge (Figure 1). Three of the women required further antibiotic therapy as there was a possible re-infection risk from an untreated sexual partner and all required advice, information and contact tracing.

An additional 22 women from the TOP group attended the GUM clinic post-operatively having received no antibiotic therapy (Figure 1). They also required advice, information and contact tracing. Therefore a total of 26 women who had undergone TOP attended the GUM department.

A variety of antibiotics were used for treatment, all of which were oral. These included for $C$. trachomatis: azithromycin $1 \mathrm{~g}$ single dose; doxycyline $100 \mathrm{mg}$ twice daily for 7 days; erythromycin $500 \mathrm{mg}$ four times daily for seven days; or erythromycin $500 \mathrm{mg}$ twice daily for 14 days; and for $N$. gonorrhoeae: ciprofloxacin $500 \mathrm{mg}$ single dose or amoxycillin $3 \mathrm{~g}$ plus probenecid $1 \mathrm{~g}$ single dose.

The longest time from TOP to any treatment, by either a gynaecologist or doctor in GUM, was 36 days. However, four women (13\%) were neither treated by the gynaecologists nor did they respond to the invitation to attend the GUM department. Subsequently, evidence of treatment for one of these women by the GP was confirmed.

The availability of results in relation to the timing of TOP was variable: 12 sets of results were reported between 1-14 days prior to the TOP; 10 sets were reported on the termination day; and the remainder after the event (Figure 2 ). The time from the initial assessment in the gynaecology clinic pre-TOP to termination varied from the same day to 17 days later, with the mode being 5 days. The majority of the TOPs (22) were carried out at 5 days or less.

Figure 2 Time from reporting results to the day of TOP

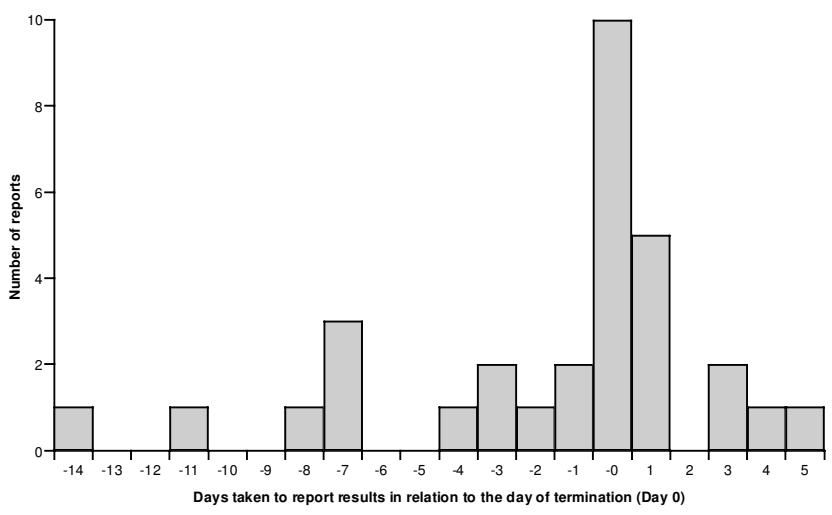

Following the TOP there were clinical signs of ascending pelvic involvement in seven of the 26 women seen in the GUM department (28\%). Six cases were identified on clinical examination in the GUM department whilst one was re-admitted 3 days post-operatively with possible retained products of conception or pelvic sepsis. None of these women had received prior antibiotic treatment.

Figure 1 Treatment location and attendance of women undergoing TOP

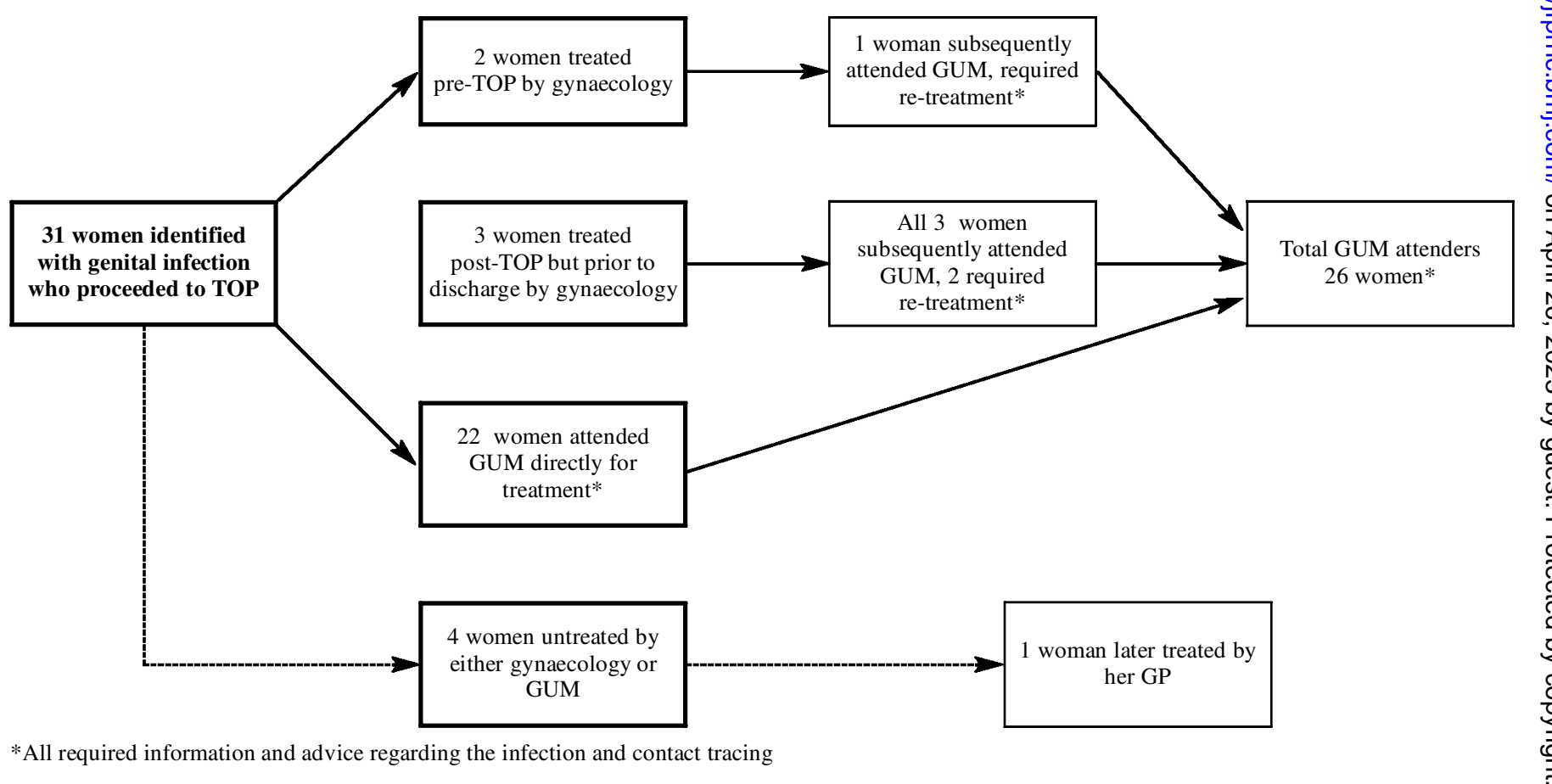


Of the 26 women: 25 were positive for $C$. trachomatis alone and one was positive for $N$. gonorrhoeae alone, and she had no evidence of pelvic infection. Hence $28 \%$ of women with chlamydial infection had clinical signs of pelvic involvement.

Twenty-six women proceeding to TOP and all nine of the women who decided to continue with the pregnancy attended the GUM department. Contact tracing was successful in $24(69 \%)$ of these 35 women.

\section{Discussion}

Consistent with others, we have identified and published data indicating a high incidence of early post-TOP complications in situations where a pre-existing genital infection, predominantly $C$. trachomatis, is present. 8,9

We advise that all women attending the gynaecology department who are booked for a TOP are offered screening for genital infection, including $N$. gonorrhoeae and $C$. trachomatis. Our overall success at screening all women and the prevalence of infection was difficult to determine for several reasons. Firstly, at the time of the study, women requesting a TOP attended general gynaecology out-patient clinics, and neither they nor their samples were identified separately as being pre-TOP. Secondly, as the operative TOP default rate was $23 \%$ analysis of operative lists was unlikely to result in a high degree of accuracy, given that many women cancel before the list is generated. Although collection of this information was not the main aim of this study, we have now instituted a system by which these data could be obtained with relative ease if required in the future.

Given our current practice a large number of women, although screened prior to TOP, do not receive appropriately timed antibiotic treatment, partly due to the short time interval between the initial assessment and operation, and partly due to the laboratory turn-around time. In our centre the latter is now being expedited due to the availability of daily assays. Further refinements are being considered, including a dedicated member of the surgical team, to ensure all positive results are noted, the delivery of sexual health advice on discharge following TOP, and periodic prospective audit at intervals to troubleshoot, if necessary.

In our previous work, using the same model of care, we identified 294 women with a genital infection who tested positive for either $C$. trachomatis, $N$. gonorrhoeae, or both, via the obstetric or gynaecology department. ${ }^{7}$ The women were tested for a variety of reasons including pre-TOP, during pregnancy, post-partum, or for presumed pelvic inflammatory disease. All were invited to attend the GUM department and $231(78.6 \%)$ did so, the majority within 2 weeks of the invitation. As a result of the initiative $73.6 \%$ of the women attending GUM had at least one sexual contact assessed and treated.

However, in this study of a separate and specific pre-TOP group, we identified a lower rate of attendance at GUM by the women identified with a genital infection (65\%). This may reflect a more difficult to reach population group or the small size of the cohort. More of the women (75\%) who had received antibiotic therapy already via the gynaecologists required re-treatment because of sexual intercourse with an untreated sexual partner than in our earlier more general work, where $41 \%$ of those already treated required retreatment. $^{7}$ The significance of this is uncertain given the small numbers in our group, but it highlights the need for advice, when giving treatment, to avoid coitus until their sexual partner has been treated. We also noted that despite using an active and direct programme for the GUM department to contact infected women and offer a 'fasttrack' appointment, four women remained untreated by either the gynaecologists or GUM. This was despite using our local strategy of three letters to each woman from GUM and a home visit. The respective gynaecologists were informed about the womens' default at GUM and untreated infection state. In addition, all of the womens' GPs were notified about the untreated infection, resulting in one further patient being treated. By the use of this safety system we have reduced as far as possible the chance of a genital infection remaining entirely untreated. However, late treatment does not protect at the vital time, during the termination, against the surgical intervention.

Almost $70 \%$ of contacts were seen and treated appropriately which, even in this difficult group, is not significantly different to our previous work. ${ }^{7}$ If contacts are not treated there is a risk of re-infection, and it is this argument which is the most cogent against a policy of giving all women anti-chlamydial treatment prior to TOP withou screening, as suggested by others. ${ }^{10}$ Otherwise the opportunity for effective contact tracing and health education will be lost. In addition, a second pelvic infection is likely to be more damaging to the woman than the initial insult. ${ }^{11}$

Of continuing concern is that women may still be missed unless screening for $C$. trachomatis is performed with nucleic acid amplification tests, as their sensitivity greatly exceeds testing methods such as culture or EIA. ${ }^{12}$ If our current policy of screening with EIA is continued we would suggest that a 'belt and braces' approach may be the best option. Treating all will allow immediate protection over the operative period and screening all will allow appropriate contact tracing and treatment. This will afford not only individual advantages, but should also reduce the community pool of infection. A more rational approach would be to use high sensitivity testing, utilising nucleic acid amplification, and treating prior to TOP all who are positive, together with active contact tracing. In the future self-sampling by women may be an option, ${ }^{13}$ although it is less important in this group as they will require clinical examination to assess gestation.

We were surprised at our findings that $23 \%$ of women who get as far as attending a gynaecology out-patient clinic, who are counselled and booked for TOP subsequently change their minds and decide to continue with the pregnancy. Our estimates for the overall operative default pattern in Nottingham vary each week, but our range is from $15-22 \%$. Hence, whilst our study group rates are comparable to the wider picture in Nottingham, they are higher than those identified by the British Pregnancy Advisory Service (BPAS) whose operative default rate is $8 \%$. This may reflect the different services available or the client base.

The timing of treatment and which antibiotic should be used are also controversial, particularly bearing in mind that almost one quarter of women requesting TOP in our cohort subsequently decided to continue with the pregnancy. It is known that azithromycin is present in the cervical mucus very soon after dosing, hence this may be a convenient, effective method if given on admission prior to TOP. ${ }^{14}$

Screening women at TOP is a valuable way in which we can identify and treat those with genital chlamydial infection, thus reducing the incidence of complications as highlighted by studies in Sweden. ${ }^{15}$ Unfortunately, in the short-term screening has cost implications which may be difficult to incorporate into limited financial budgets. However, given the longer term financial savings for the NHS together with the reductions in psychological and physical morbidity to the woman and her family, it would seem short-sighted not to invest in prevention now. $1,16-18$ 


\section{Conclusion}

Screening and treatment for genital infection prior to TOP provides a valuable opportunity to preserve the future sexual health of women. However, it is essential to organise our resources so that treatment is given before surgical intervention. In addition, appropriate sexual contact tracing and treatment is essential, otherwise the woman will be at risk of re-infection and the community pool of infection will not be reduced.

\section{Statements on funding and competing interests}

Funding. None.

Competing interests. None.

References

Blackwell AL, Thomas PD, Wareham K, et al. Health gains from screening for infection of the lower genital tract in women attending for termination of pregnancy. Lancet 1993; 342: 206-210. Stray-Pedersen B, Biornstad J, Dahl M, et al. Induced abortion: microbiological screening and medical complications. Infection 1991; 19: 305-308.

3 Sorensen JL, Thranov I, Hoff G, et al. Early and late-onset pelvic inflammatory disease among women with cervical Chlamydia trachomatis infection at the time of induced abortion-a follow-up study. Infection 1994; 22: 242-246.

Chow JM, Yonekura ML, Richwald GA, et al. The association between Chlamydia trachomatis and ectopic pregnancy. A matched pair, case-control study. JAMA 1990; 263: 3164-167.
5 Zana J, Thomas D, Muffat-Joly M, et al. An experimental model for salpingitis due to Z 5: $274-278$

6 Penney GC. Prophylactic antibiotic therapy for abortion. In: Templeton A, editor. The prevention of pelvic infection. London: RCOG Press, 1996: 211-222.

Haddon L, Heason J, Fay T, et al. Managing STIs identified after testing outside genitourinary medicine departments: One model of care. Sex Transm Inf 1998; 74: 256-257. infective complications of induced abortion (letter). Br J Obstet Gynaecol 1999; 106: 287. Osser S, Persson K. Postabortal infectious morbidity after antibiotic treatment of chlamydiapositive patients. Sex Transm Dis 1989; 16: 84-87.

10 Penney GC, Thomson M, Norman J, et al. A randomised comparison of strategies for reducing infective complications of induced abortion. Br J Obstet Gynaecol 1998; 105: 599-604.

1 Patton DL, Wolner-Hanssen P, Zeng W, et al. The role of spermatozoa in the pathogenesis of Chlamydia trachomatis salpingitis in a primate model. Sex Transm Dis 1993; 20: 214-219.

12 Young H, Moyes A, Horn K, et al. PCR testing of genital and urine specimens compared with culture for the diagnosis of chlamydial infection in men and women. Int J STD \& AIDS 1998 9: 661-665.

13 Polaneczky M, Quigley C, Pollock L, et al. Use of self-collected vaginal specimens fo detection of Chlamydia trachomatis infection. Obstet Gynaecol 1998; 91: 375-378.

14 Worm A-M, Osterlind A. Azithromycin levels in cervical mucus and plasma after a single $1.0 \mathrm{~g}$ oral dose for chlamydial cervicitis. Genitourin Med 1995; 71: 244-246.

Egger M, Low N, Smith G D, et al. Screening for chlamydial infections and the risk of ectopic pregnancy in a county in Sweden: ecological analysis. Br Med J 1998; 316: 1776-1780.

16 Trachtenberg A, Washington AE, Halldorson S. A cost-based decision analysis for Chlamydia screening in California family planning clinics. Am J Obstet Gynecol 1988; 71: 101-108.

7 Genc M, Mardh P-A. A cost-effectiveness analysis of screening and treatment for Chlamydic

18 Howell AR, Quinn TC, Gaydos CA. Screening for Chlamydia trachomatis in asymptomatic women attending family planning clinics. A cost-effectiveness analysis of three strategies. An Intern Med 1998; 128: 277-284. 